\title{
Fast modelling and simulation of the dynamic behaviour of a wet multidisc clutch during the engagement phase
}

\author{
Nikolaos Rogkas ${ }^{1, *}$, Georgios Vasileiou ${ }^{1}$, Efstratios Tsolakis ${ }^{1}$, Vasileios Spitas ${ }^{1}$, and Pavlos Zalimidis ${ }^{2}$ \\ ${ }^{1}$ National Technical University of Athens, School of Mechanical Engineering, Laboratory of Machine Design, 9 Iroon Polytechneiou, \\ 15780 Athens, Greece \\ ${ }^{2}$ School of Pedagogical and Technological Education, Department of Mechanical Engineering Educators, Iraklio Attikis, 14121 Athens, \\ Greece
}

\begin{abstract}
In recent years multidisc wet friction clutches are of great importance to manufacturers of automatic transmissions (ATs) for the automotive industry, particularly since the introduction of double-clutched ATs. Their main advantage compared to their dryfriction counterparts is that they ensure smooth engagement, high reliability and long service life. Their progressive engagement due to the developed Couette flow between the discs enables them to be used both as clutches and as brakes in order to control power flow in simultaneously engaged geared shafts in the AT. Due to the coupled nature between the mechanical and the fluid dynamics regimes governing their operation, these systems are highly complex to be treated analytically and instead numerical approaches have proven to provide better results. However, the numerical treatment of such problems provides only case-specific results, which cannot be generalised and are not able to provide a general insight in the complex dynamics of the device. Furthermore the computational cost and the associated modelling and simulation effort during the design phase is high, making the incorporation of such methods in iterative design processes and algorithms counterproductive. In this paper the modelling of the dynamic behaviour of a wet multidisc clutch during the engagement phase is performed, via the combination of analytical and numerical methods and conclusions are drawn about the effect of the main geometric, kinematic and dynamic design parameters on the clutch's response. The dynamic modelling is performed by applying the principle of linear and angular momentum on each disc. The effect of the fluid film is taken into account through the solution of the governing Navier-Stokes equations via CFD analysis or by the use of semianalytical solutions with high accuracy, where applicable. Therefore both the developed pressure field and the torque of the fluid film are calculated efficiently and used in the simulation of the system. The flow is assumed to be laminar and the discs rigid and flat.
\end{abstract}

\section{Introduction}

Successful modelling and evaluation of a wet multidisc clutch is a fairly demanding and complicated process if treated analytically and thus numerical methods are employed to solve the coupled problem of solid and fluid mehcanics. A simplified configuration of a wet multiplate clutch is presented in Fig. 1 where the following two systems are indetified: the primary system consisting of the clutch input shaft engaged with the engine and the male discs and the secondary system consisting of the output shaft engaged with the housing and the female disks. The progressive engagement of the clutch due to the developed shear stresses applied by the Couette flow on the surface of the discs, implies the radial outflow of the fluid (squeeze effect) which is provided by the axial displacement of discs by means of a hydraulic actuator. As a result of this hydrodynamic transmission, the film thickness between the discs decreases and the output torque of the clutch builds up to the torque required by the load, while also the input and output shafts are synchronized at the same speed.
Regarding the modelling of the clutch dynamic behaviour during the engagement phase one has to predict

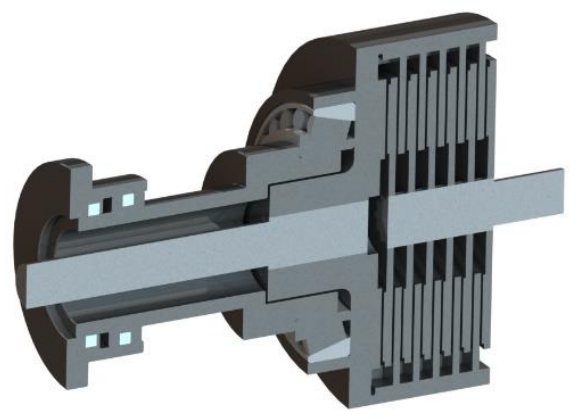

Fig. 1. CAD model of Wet Multidisc Clutch.

accurately the developed pressure and velocity field distributions within the film between two consecutive discs. This is usually performed by applying the necessary CFD analysis to solve the governing flow equations. The numerical treatment of the flow dynamics problem is very common and effective and leads to the conclusion of case-spesific results about the effect of a 
variety of design parameters on the reponse of the clutch [1-4]. On the other hand, the incorporation of such methods in the modelling of complete power transmission systems involving wet multidisc clutches [5], [6] (such us the well-known double clutch ATs) makes the total simulation proccess of the power-train consuming in terms of time and recourses. Aiming in the composition of wet clutch fast models that assist the design process in terms of computational cost, this paper proposes the use of semi-analytical methods in order to study the dynamic behavior of a multidisc wet clutch during the engagement phase.

\section{Analytical modeling}

In recent years, the necessity of better-understanding the physics of the fluid mechanics problem as well as the need for the extraction of fast generic results, has led many researchers into putting efforts in deriving analytical solutions of the similar-type flow problems [79]. Those efforts are concentrated in deriving truncated forms of the Navier Stokes equations set that are analytically solvable. The basis of this approach is the problem of the flow between rotating discs as initially studied by v. Karman, in the form of one infinite disk rotating at a constant angular velocity in fluid which is initially at rest. Currently, similar models based on simplification of the flow equations are abailable, nevertheless they are applied mainly on v. Karman type of flows problems and they do not take into account the squeezing effect that exists in a wet clutch. Additionally the necessary truncations made, to achieve an analytical solution procedure, constrain the validity of the obtained expressions, to certain range of the operation envelope. A simplified schematic representation of the problem of two consecutive discs inside a wet clutch is presented in Fig. 2.

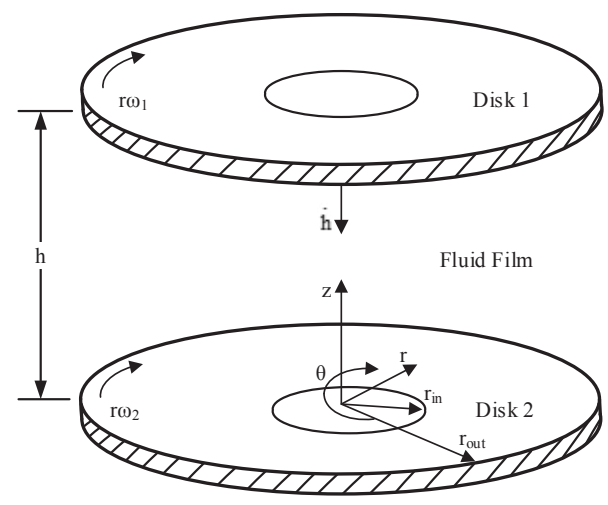

Fig. 2. Wet clutch-2 discs model.

The flow is considered to be steady, laminar, incompressible [10], isothermal, axisymmetric and the fluid is newtonic, so that: $\rho=$ const, $\mu=$ const. The velocity vector in cylindrical coordinates is: $\boldsymbol{u}=$ $\left[\begin{array}{lll}u_{r} & u_{\vartheta} & u_{z}\end{array}\right]$ and the continuity and Navier-Stokes equations in their conservative form are respectively:

$$
\begin{gathered}
\boldsymbol{\nabla u}=0 \\
\frac{\partial \boldsymbol{u}}{\partial t}+(\nabla \boldsymbol{u}) \boldsymbol{u}=\frac{1}{\rho} \nabla P+\nu \nabla^{2} \boldsymbol{u}
\end{gathered}
$$

Performing the appropriate order of magnitude analysis in Eqs. (1) and (2), so that terms with insignificant magnitude order can by neglected, they reduce to $[+++]$ :

$$
\begin{gathered}
-\frac{u_{\theta}^{2}}{r}=-\frac{1}{\rho} \frac{\partial P}{\partial r}+\frac{\mu}{\rho} \frac{\partial^{2} u_{r}}{\partial z^{2}} \\
\frac{\partial^{2} v_{\theta}}{\partial z^{2}}=0 \\
\frac{\partial P}{\partial z}=0
\end{gathered}
$$

And due to the axi-symmetry of the problem the continuity equation becomes:

$$
\frac{1}{r} \frac{\partial\left(r v_{r}\right)}{\partial r}+\frac{\partial v_{z}}{\partial z}=0
$$

The boundary conditions of the problem are:

$$
\begin{gathered}
P\left(r_{\text {in }}\right)=P\left(r_{\text {out }}\right)=0 \\
u_{z}(r, h)=\dot{h} \text { and } u_{z}(r, 0)=0 \\
u_{r}(r, h)=0 \text { and } u_{r}(r, 0)=0 \\
u_{\theta}(r, h)=\omega_{1} r \text { and } u_{\theta}(r, h)=\omega_{2} r
\end{gathered}
$$

The solution of Eqs. (3) and (5) using the boundary conditions (9) and (10) is:

$$
\begin{gathered}
v_{r}(r, z)=\frac{1}{2 \mu} \frac{\mathrm{d} z}{\mathrm{~d} r} z(z-h)- \\
\frac{\rho r z^{2}}{\mu}\left(\frac{1}{2} \omega_{2}^{2}+\frac{z}{3 h} \omega^{2} \Delta \omega+\frac{z^{2}}{12 h^{2}} \Delta \omega^{2}\right) \\
v_{\theta}(r, z)=r\left(\omega_{2}+\frac{z}{h} \Delta \omega\right)
\end{gathered}
$$

where $\Delta \omega=\omega_{1}-\omega_{2}$. Substituting Eq. (11) into Eq. (6) and integrating with respect to $\mathrm{z}$ under the boundary conditions (8) yields the second order differential equation for pressure:

$$
\frac{\mathrm{d}^{2} P}{\mathrm{~d} r^{2}}+\frac{1}{r} \frac{\mathrm{d} P}{\mathrm{~d} r}=\frac{12 \mu \dot{h}}{h^{3}}+2 \rho\left(2 \omega_{2}^{2}+\omega_{2} \Delta \omega+\Delta \omega^{2}\right)
$$

Integrating Eq. (12) under the boundary conditions (7) the pressure distribution versus the radius is obtained:

$$
\begin{gathered}
\mathrm{P}(\mathrm{r})=\left[\frac{\rho}{2}\left(\omega_{2}^{2}+\Delta \omega \omega_{2}+\frac{3}{10} \Delta \omega^{2}\right)+3 \frac{\mu h}{h^{3}}\right] \times \\
{\left[r^{2}-r_{\text {in }}^{2}+\left(r_{\text {out }}^{2}-r_{\text {in }}^{2}\right) \frac{\ln \left(\frac{r}{r_{\text {in }}}\right)}{\ln \left(\frac{r_{\text {in }}}{r_{\text {out }}}\right)}\right]}
\end{gathered}
$$


Finally the axial force due to fluid pressure and the torque due to the fluid shear stress on the discs are retrieved.

$$
\begin{gathered}
\mathrm{F}_{\text {fluid }}=2 \pi \int_{r_{\text {in }}}^{r_{\text {out }}} P(r) r \mathrm{~d} r \\
\mathrm{~T}=2 \pi \mu \int_{r_{\text {in }}}^{r_{\text {out }}} r^{2} \frac{\partial u_{\theta}(r, z)}{\partial z} \mathrm{~d} r
\end{gathered}
$$

Regardless of the simplifications made for obtaining the analytical expressions of pressure and torque they can still be used to provide acceptable accuracy throughout the operational envelope of the clutch, especially at the engagement phase where the film thickness becomes sufficiently small compared to the nominal size of the gap. The accuracy of the analytical expressions is compared to the results of a CFD model and found to be satisfactory regarding the calculation of the necessary flow parameters.

\section{CFD model setup}

Besides the above analytical approach, in the present study two CFD models are constructed using the ANSYS Fluent software. This is performed in order to examine the accuracy of Eqs. (15) and (18) and to draw conclusions about the influence of the radial clearance on the developed pressure field. The flow is considered steady state, laminar and axisymmetric including swirl. The geometry of the models is presented in Figs. (3-a), (3-b) and the boundary conditions hold from Eqs. (7) to (10).

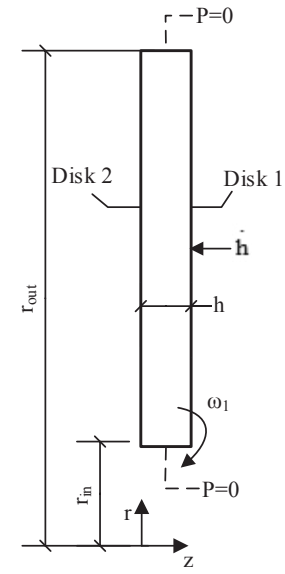

(a)

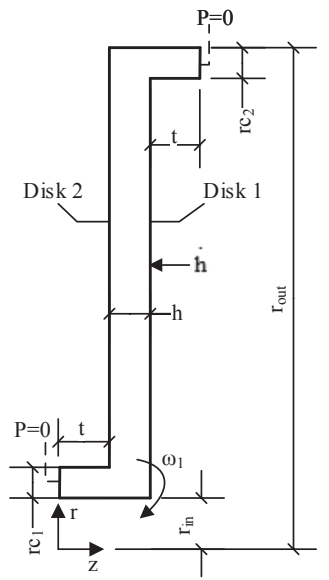

(b)
Fig. 3. Geometry and boundary conditions of 2-discs CFD model - (a) without a radial clearance, (b) including a radial clearance.
The parameters are:

Table 1. Constant parameters values

\begin{tabular}{cll}
\hline \hline $\mathrm{r}_{\text {out }}$ & 57.5 & {$[\mathrm{~mm}]$} \\
$\mathrm{r}_{\text {in }}$ & 20 & {$[\mathrm{~mm}]$} \\
$\mathrm{t}$ & 3 & {$[\mathrm{~mm}]$} \\
$\omega_{2}$ & 0 & {$[\mathrm{rpm}]$} \\
$\mu$ & 0.01 & {$[\mathrm{~Pa} \mathrm{~s}]$} \\
$\rho$ & 800 & {$\left[\mathrm{~kg} / \mathrm{m}^{3}\right]$} \\
\hline
\end{tabular}

As previously mentioned, Eqs. (14) and (12) hold a sufficient accuracy as the gap between two consecutive discs tends to decrease, which can be observed in Figs. (4-a) to (4-c) and (5).

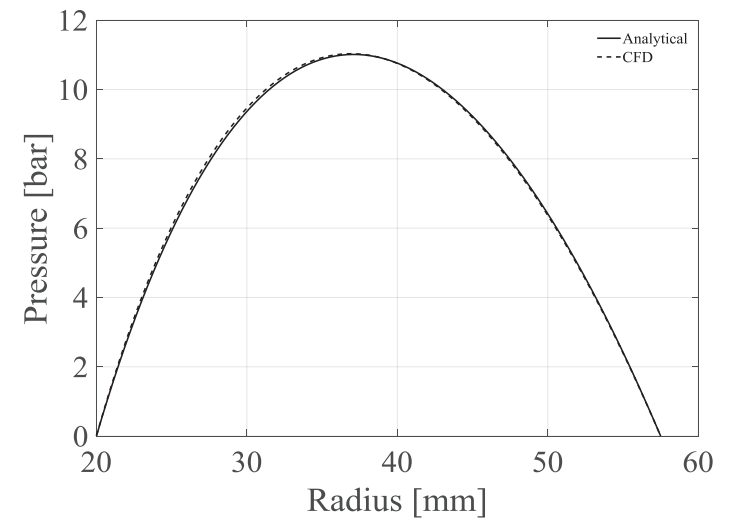

Fig. 4-a. Pressure distribution versus radius. Comparison between analytical approach (Eq. 14) and CFD results for $\mathrm{h}=0.1 \mathrm{~mm}, \dot{\mathrm{h}}=0.05 \mathrm{~m} / \mathrm{s}$ and $\omega=5000 \mathrm{rpm}$.

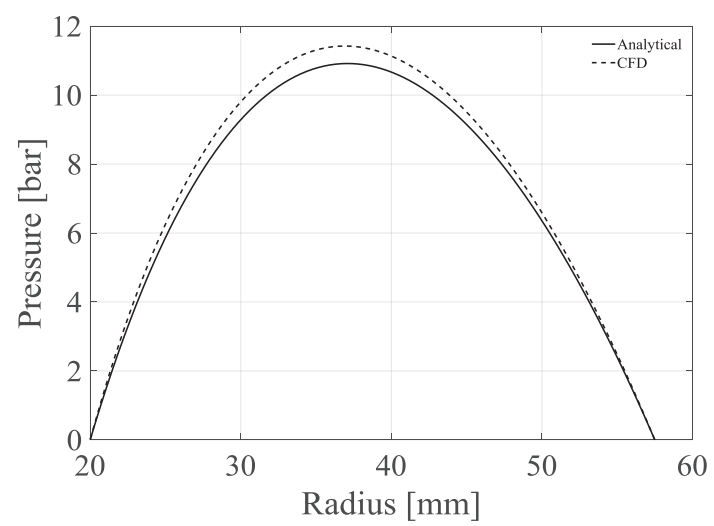

Fig. 4-b. Pressure distribution versus radius. Comparison between analytical approach (Eq. 14) and CFD results for $\mathrm{h}=0.1 \mathrm{~mm}, \dot{\mathrm{h}}=0.05 \mathrm{~m} / \mathrm{s}$ and $\omega=3000 \mathrm{rpm}$. 


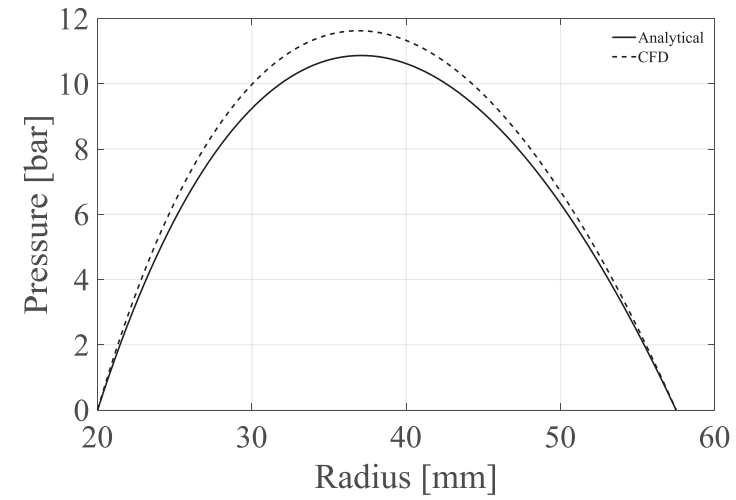

Fig. 4-c. Pressure distribution versus radius. Comparison between analytical approach (Eq. 14) and CFD results for $\mathrm{h}=0.1 \mathrm{~mm}, \dot{\mathrm{h}}=0.05 \mathrm{~m} / \mathrm{s}$ and $\omega=1000 \mathrm{rpm}$.

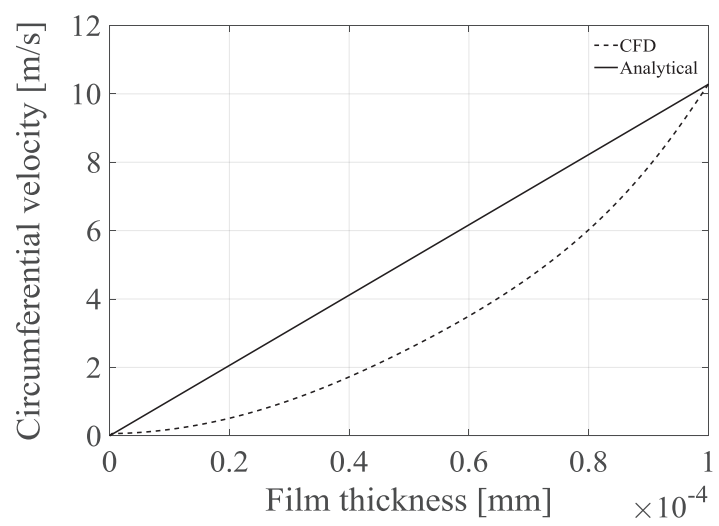

Fig. 5. Circumferential velocity $\mathrm{u}_{\theta}$ versus film thickness $h$. Comparison between analytical approach(Eq. 12) and CFD results for $\mathrm{h}=0.1 \mathrm{~mm}, \dot{\mathrm{h}}=0.05 \mathrm{~m} / \mathrm{s}$ and $\omega=5000 \mathrm{rpm}$.

In Fig. (6) the velocity streamlines are presented.
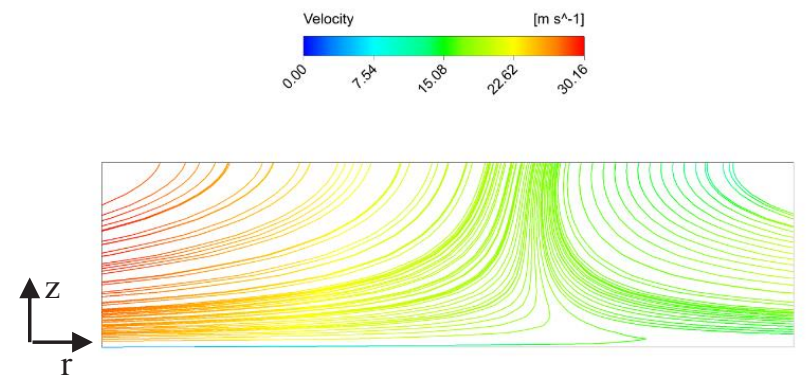

Fig. 6. Velocity streamlines referring to the geometry of Fig. (3-a). $\mathrm{h}=0.1 \mathrm{~mm}, \dot{\mathrm{h}}=0.05 \mathrm{~m} / \mathrm{s}$ and $\omega=5000 \mathrm{rpm}$.

Regarding the radial clearance $r_{c}$, it affects the developed pressure in a major way as it acts either a resistance or a relief to the flow, depending on its value. Due to the fact that its influence cannot be incoporated in the analytical approach, the models of Figs. (3-a) and (3-b) will be used accordingly. If $\bar{P}_{n o m}$ and $\bar{P}_{r c}$ are the the average pressure values respectively and for simplicity $r_{c 1}=r_{c 2}=r_{c}$ then it is assumed that:

$$
\frac{\bar{P}_{r c}}{\bar{P}_{n o m}}=\delta\left(r_{C}, h, \dot{h}, \omega_{1}\right)
$$

Therefore the ratio $\delta$ is a function of four variables and its total differential is:

$$
\mathrm{d} \delta\left(r_{C}, h, \dot{h}, \omega_{1}\right)=\frac{\partial \delta}{\partial r_{c}} \mathrm{~d} r_{c}+\frac{\partial \delta}{\partial h} \mathrm{~d} h+\frac{\partial \delta}{\partial \dot{h}} \mathrm{~d} h+\frac{\partial \delta}{\partial \omega_{1}} \mathrm{~d} \omega_{1}
$$

The further parametric investigation of Eq. (18) proceeds via the examination of the dependence of $\delta$ on $\dot{\mathrm{h}}$ and $\omega_{1}$. This is achieved by choosing multiple design points, thus forming suitable combinations of the variables $h, \dot{h}, \omega_{1}, r_{c}$ and finally performing the CFD simulations based on the geometry of the models of Figs. $(3-a),(3-b)$. The results are presented in the figures below.

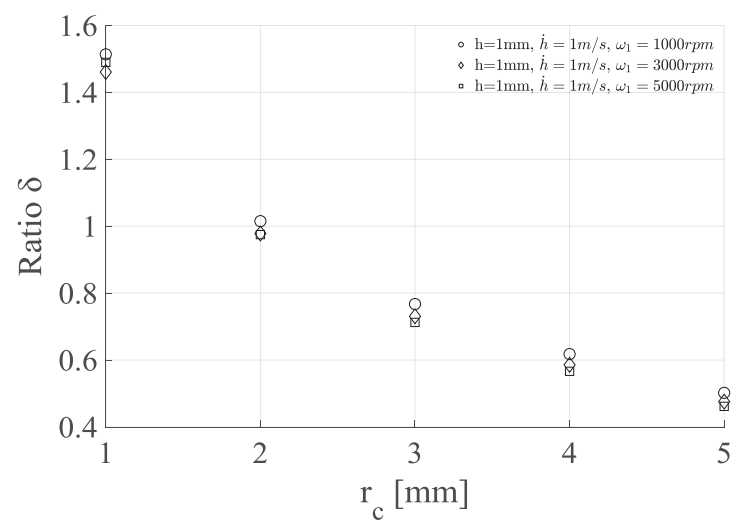

Fig. 7-a. Ratio $\delta$ versus radial clearance, design point 1 .

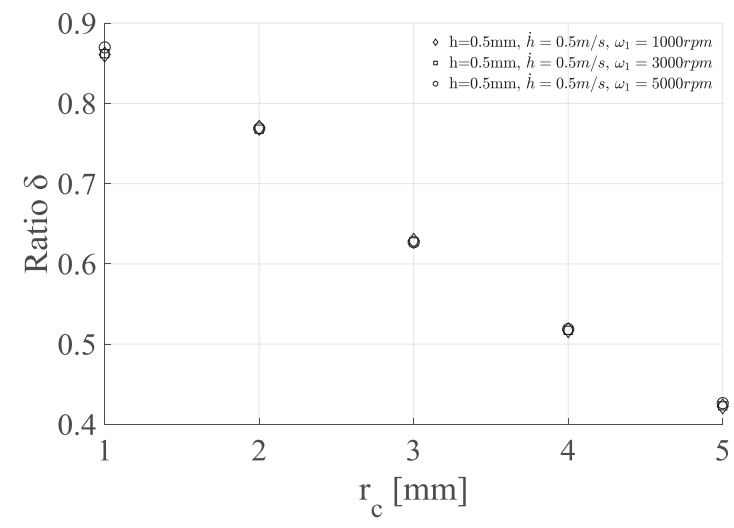

Fig. 7-b. Ratio $\delta$ versus radial clearance, design point 2.

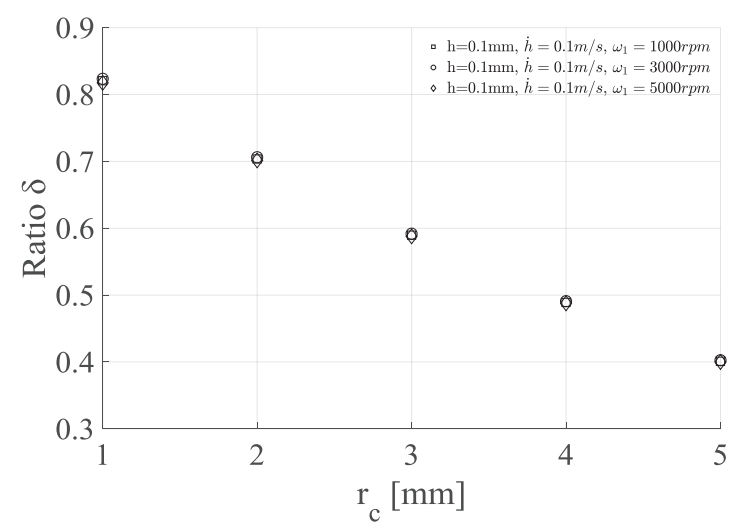

Fig. 7-c. Ratio $\delta$ versus radial clearance, design point 3 . 
Observing the Figs. (7) to (8) it is concluded that the dependence of $\delta$ on $\dot{\mathrm{h}}$ and $\omega_{1}$ is negligible and therefore $\delta_{\omega l}=\delta_{\dot{h}} \approx 0$.

$$
d \delta\left(r_{C}, h\right)=\frac{\partial \delta}{\partial r_{c}} d r_{c}+\frac{\partial \delta}{\partial h} d h
$$

The dependence of $\delta$ on $r_{c}$ may be approximated by the equation:

$$
\delta\left(r_{c}\right)=e^{b r_{c}}
$$

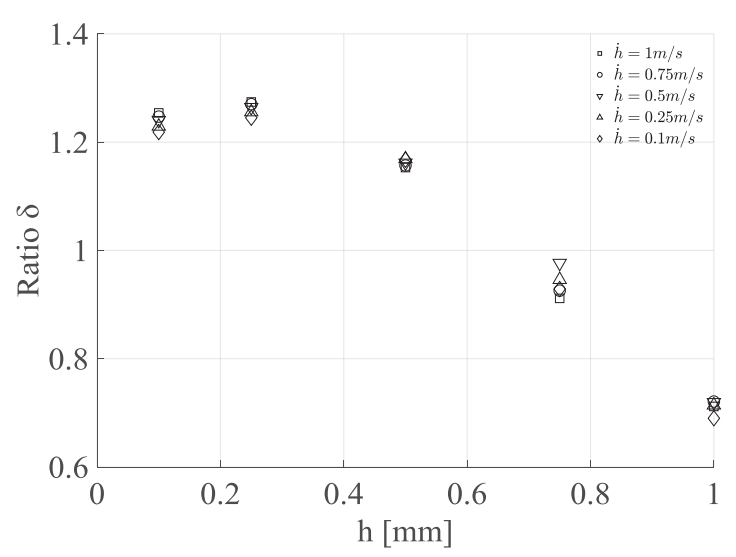

Fig. 8. Ratio $\delta$ versus film thickness for varying closing velocities and constant radial clearance $r_{c}=1 \mathrm{~mm}$.

and constant $\mathrm{b}$ can be calculated after fitting the data in Fig. (8), therefore:

$$
\delta(1)=-0.5897 \mathrm{~h}^{1.91}+1.288
$$

from which the final empirical formula is obtained:

$$
\delta\left(r_{C}, h\right)=\left(-0.5897 h^{1.91}+1.288\right)^{r_{C}}
$$

\section{Dynamic modelling}

Afterwards the semi-analytical treatment of the flow problem between two consecutive discs of the clutch is investigated, thus the integration of the obtained solutions in the total dynamic model of the clutch remains yet unexplored. The first step to approach the system of a wet multidisc wet clutch is to integrate the solutions for the two-discs system Fig. (2) into a multidisc wet clutch model that is fed with dynamic and kinematic excitations of a power transmission system. Such a simplified model is presented in Fig (9). The inertia of the other parts of the clutch is neglected.

Let the total number of discs (both driving and driven) be $n$ (driving discs: $i=1,3, \ldots, n-1$ and driven discs: $\mathrm{i}=2,4, \ldots, n)$ and it is assumed that the actuator force $F_{\text {app }}$ and torque $\mathrm{T}_{\text {app }}$ are exerted on the top disc ( $\mathrm{i}=1$ ) while the pressure plate force $\mathrm{F}_{\text {stop }}$ and the torque load $\mathrm{T}_{\text {load }}$ are exerted on the bottom disc $(i=n)$.
The total torque transmitted to the output shaft will be the sum of the torque developed on each of the driven discs.

$$
T_{\text {total }}=\sum_{\mathrm{i}=1}^{\mathrm{i}=\mathrm{n}} T_{\mathrm{i}}, \quad \mathrm{i}=2,4, \ldots, \mathrm{n}
$$

The DOFs of the model are:

$$
\boldsymbol{y}=\left[\theta, \mathrm{z}_{1}, \mathrm{z}_{2}, \ldots, \mathrm{z}_{\mathrm{n}}\right]
$$

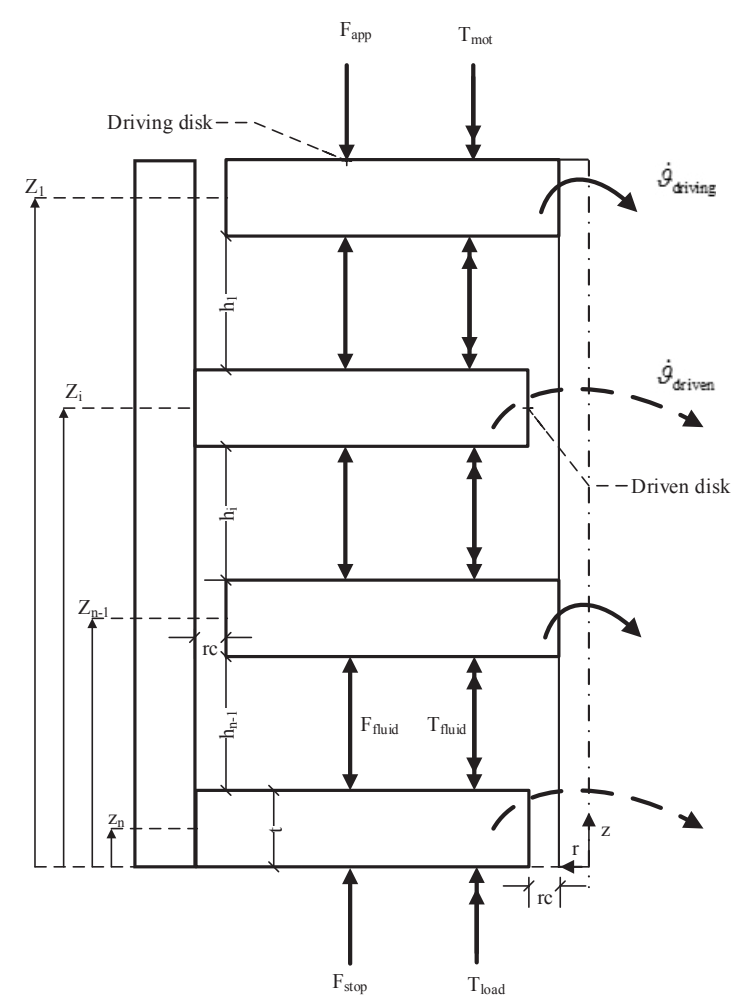

Fig. 9. Multidisc wet clutch simplified model

Calculating $\mathrm{F}_{\text {fluid }}$ and $\mathrm{T}_{\text {fluid }}$ from Eqs. (15) and (16) respectively, gives:

$$
\mathrm{F}_{\text {fluid }}=\mathrm{r}_{\mathrm{a}}\left(\frac{3 \mu \dot{h}}{h^{3}}+\frac{\rho}{2}\left(2 \omega_{2}^{2}+\omega_{2} \Delta \omega+\Delta \omega^{2}\right)\right)
$$

where

$$
\begin{gathered}
r_{a}=\frac{\pi}{2}\left(r_{\text {in }}^{2}-r_{\text {out }}^{2}\right) \frac{\ln \left(\frac{r_{\text {in }}}{r_{\text {out }}}\right)\left(r_{\text {in }}^{2}-r_{\text {out }}^{2}\right)+r_{\text {out }}^{2}-r_{\text {in }}^{2}}{\ln \left(\frac{r_{\text {in }}}{r_{\text {out }}}\right)} \\
T_{\text {fluid }}=\frac{\pi}{2} \mu \Delta \omega\left(r_{\text {out }}^{4}-r_{\text {in }}^{4}\right)
\end{gathered}
$$

Applying the linear momentum conservation theorem at each disc, one gets the following set of non linear 
differential equations describing the axial displacement of each disc:

disc \#:1 (driving disc)

$$
\begin{gathered}
\ddot{z}_{1}=-\frac{1}{m} F_{\text {app }}+\frac{1}{m} r_{a} \times \\
{\left[3 \mu \frac{\dot{z}_{1}-\dot{z}_{2}}{\left(z_{1}-z_{2}-\mathrm{t}\right)^{3}}+\frac{\rho}{2} \mathrm{f}\left(\dot{\theta}, \dot{\theta}_{\text {app }}\right)\right] \times \delta\left(1, z_{1}-z_{2}-t\right)}
\end{gathered}
$$

disc \#: i (driving or driven disc)

$$
\begin{gathered}
\ddot{z}_{i}=\frac{r_{a}}{m} \times-\left\{\left[3 \mu \frac{\dot{z}_{\mathrm{i}-1}-\dot{z}_{i}}{\left(\dot{z}_{\mathrm{i}-1}-\dot{z}_{i}-t\right)^{3}}+\frac{\rho}{2} \mathrm{f}\left(\dot{\theta}, \dot{\theta}_{a p p}\right)\right] \times \delta\left(1, z_{\mathrm{i}-1}-z_{\mathrm{i}}-t\right)\right. \\
\left.\left[3 \mu \frac{\dot{z}_{i}-\dot{z}_{\mathrm{i}+1}}{\left(\dot{z}_{i}-\dot{z}_{i+1}-t\right)^{3}}+\frac{\rho}{2} \mathrm{f}\left(\dot{\theta}_{a p p}, \dot{\theta}\right)\right] \times \delta\left(1, z_{\mathrm{i}}-z_{\mathrm{i}+1}-t\right)\right\}
\end{gathered}
$$

$\underline{\text { disc \#: } \mathrm{n} \text { (fixed disc) }}$

$$
\ddot{z}_{i}=\dot{z}=0, z=\mathrm{const}
$$

where

$$
\mathrm{f}\left(\dot{\theta}, \dot{\theta}_{\text {app }}\right)=2 \dot{\theta}^{2}+\dot{\theta}\left(\dot{\theta}_{a p p}-\dot{\theta}\right)+\left(\dot{\theta}_{a p p}-\dot{\theta}\right)^{2}
$$

The torque on each disc is calculated:

$$
T_{i}=\left\{\begin{array}{lc}
\left.T_{\text {fluid }}\right|_{h_{i}}-T_{\text {load }}, & i=1 \\
\left.T_{\text {fluid }}\right|_{h_{i-1}}-\left.T_{\text {fluid }}\right|_{h_{i},}, & 1<i<n \\
\left.T_{\text {fluid }}\right|_{h_{i}}-T_{\text {load }}, & i=n
\end{array}\right.
$$

Finally applying the conservation of angular momentum for the system of the discs one gets:

$$
T_{\text {total }}=\sum_{\mathrm{i}=1}^{\mathrm{i}=\mathrm{n}} I_{\mathrm{i}} \dot{\theta}, \quad \mathrm{i}=2,4, \ldots, \mathrm{n}
$$

where $I_{\mathrm{i}}$ the rotational inertia of each driven disc. Combining Eqs. (23), (26), (30) and (31) the diferrential equation for $\dot{\theta}$ is obtained:

$$
\ddot{\theta}=\frac{\pi}{2} \frac{r_{\text {out }}{ }^{4}-r_{\text {in }}{ }^{4}}{\frac{n}{2} I}\left(\dot{\theta}_{\text {app }}-\dot{\theta}\right) \sum_{i=1}^{i=n-1} \frac{1}{z_{i}-z_{i+1}-t}-\frac{1}{\frac{n I}{2}} T_{\text {load }}
$$

The equations (27), (28), (29) are (32) are solved numerically using the function ode45 in Matlab and the results are presented below using the values of Table 2 .

Table 2. Constant parameters values.

\begin{tabular}{cll}
\hline \hline $\mathrm{n}$ & 4 & \\
$t$ & 3 & {$[\mathrm{~mm}]$} \\
$r_{c}$ & 1 & {$[\mathrm{~mm}]$} \\
$F_{\text {app }}$ & 0.1 & {$[\mathrm{kN}]$} \\
$\dot{\theta}_{\text {app }}$ & $3000[\mathrm{rpm}]$ \\
$T_{\text {load }}$ & 50 & {$[\mathrm{Nm}]$} \\
\hline \hline
\end{tabular}

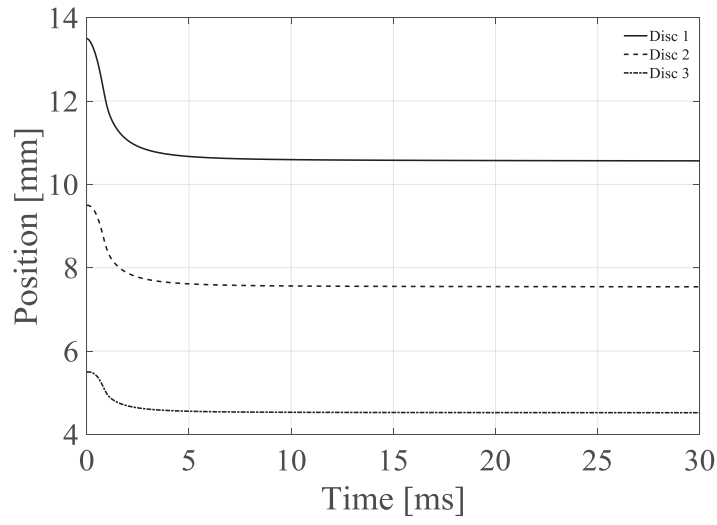

Fig. 10. Discs position versus time.

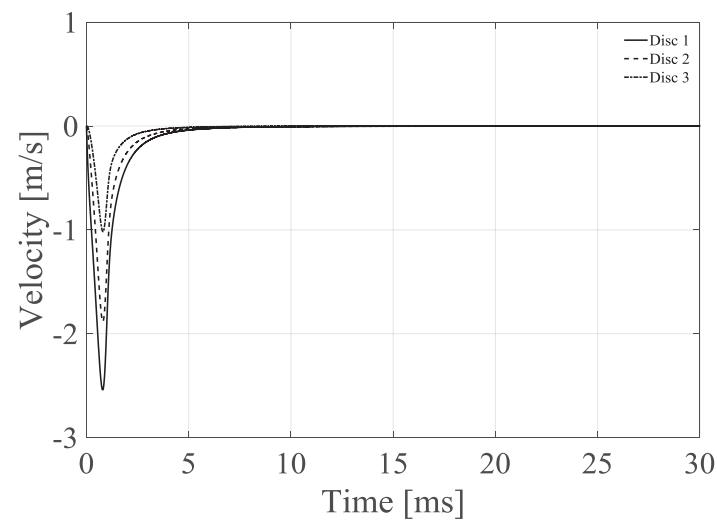

Fig. 11. Discs velocity versus time.

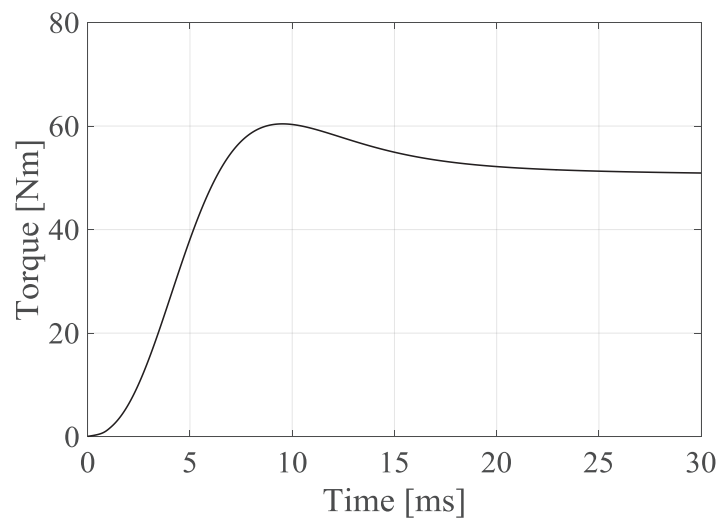

Fig. 12. Motor load versus time.

The simulations above were carried in a middle-level PC with $16 \mathrm{~Gb}$ of RAM, and the total simulation time was $\sim 10 \mathrm{~min}$. This is considerably smaller than the corresponding time required to perform the same simulation using a CFD analysis on the dynamic model of Fig. 8 instead. 


\section{Conclusions}

In this paper, a semi-analytical procedure is proposed in order to study the dynamic behaviour of a multidisc wet clutch during the hydrodynamic engagement phase. This is achieved by obtaining a set of analytical solutions regarding a truncated set of the governing flow equations. Afterwards the expressions are used in a simplified model of the clutch that includes the rotational and axial displacement of the discs, which, if simulated accordingly, can yield results over a wide range of geometric, kinematic and dynamic parameters of the clutch. The lack of accuracy is counterbalanced by the fast convergence of the dynamic model.

This research is co-financed by Greece and the European Union (European Social Fund- ESF) through the Operational Programme «Human Resources Development, Education and Lifelong Learning» in the context of the project "Strengthening Human Resources Research Potential via Doctorate Research" (MIS-5000432), implemented by the State Scholarships Foundation (IKY).

\section{References}

1. A. Bassi, M. Milani, L. Montorsi, S. Terzi (2016). Dynamic analysis of the lubrication in a wet clutch of a hydromechanical variable transmission. SAE International Journal of Commercial Vehicles, 9(2016-01-8099), 280-290.

2. Y. Yuan, P. Attibele, Y. Dong (2003). CFD simulation of the flows within disengaged wet clutches of an automatic transmission (No. 2003-010320). SAE Technical Paper.

3. S. Sfarni, E. Bellenger, J. Fortin, M. Malley (2011). Numerical and experimental study of automotive riveted clutch discs with contact pressure analysis for the prediction of facing wear. Finite Elements in Analysis and Design, 47(2), 129-141.

4. M. M. Razzaque, T. Kato (1999). Effects of groove orientation on hydrodynamic behavior of wet clutch coolant films. Journal of tribology, 121(1), 56-61.

5. Y. Yang, R. C. Lam, T. Fujii (1998). Prediction of torque response during the engagement of wet friction clutch. SAE transactions, 1625-1635.

6. M. Barr, K. Srinivasan (2015). Estimation of wet clutch friction parameters in automotive transmissions (No. 2015-01-1146). SAE Technical Paper.

7. C. R. Aphale, J. Cho, W. W. Schultz, S. L. Ceccio, T. Yoshioka, H. Hiraki (2006). Modeling and parametric study of torque in open clutch plates. Journal of tribology, 128(2), 422-430.

8. S. Iqbal, F. Al-Bender, B. Pluymers, W. Desmet, (2013). Mathematical model and experimental evaluation of drag torque in disengaged wet clutches. ISRN Tribology, 2013.

9. Y. Yuan, E. A. Liu, J. Hill, Q. Zou (2007). An improved hydrodynamic model for open wet transmission clutches. Journal of Fluids Engineering, 129(3), 333-337.
10. J. Huang, J. Wei, M. Qiu (2012). Laminar flow in the gap between two rotating parallel frictional plates in hydro-viscous drive. Chinese Journal of Mechanical Engineering, 25(1), 144-152. 